\title{
Plasma Process Modeling for Integrated Circuits Manufacturing
}

\author{
M. MEYYAPPAN and T.R. GOVINDAN \\ Scientific Research Associates, Inc. P.O. Box 1058 Glastonbury, CT 06033
}

\begin{abstract}
A reactor model for plasma-based deposition and etching is presented. Two-dimensional results are discussed in terms of plasma density, ion flux, and ion energy. Approaches to develop rapid CAD-type models are discussed.
\end{abstract}

Keywords: plasma deposition, plasma etching, modeling, reactor model

\section{INTRODUCTION}

Plasma etching and deposition are key steps in device processing. The decreasing feature size of devices places a stringent demand on these processes in terms of critical dimension control, uniformity, and selectivity. Process optimization to achieve the desirable goals can benefit from models in a computer-aided design (CAD) environment. The models must be able to predict growth/etch rate, film composition, uniformity, etc. Etch or deposition profile shape is also of interest, for which programs such as EVOLVE, SPEEDIE, and SIMBAD are available. These programs need information on neutral and ionic species flux, ion energy, ion angle of incidence, etc. To obtain such data, one needs to model the process at the reactor level.

\section{MODEL}

The details of the model have been published [1] and only a brief outline is given below. The transport of electrons and ions is described by the moments of the
Boltzmann equation representing conservation of mass, momentum, and energy. The neutral gas velocity is small compared to that of electrons and ions; the latter two are primarily lost at the electrodes. So, the transport of electrons and ions is considered in a stationary, constant density, background gas. The mass conservation equation consists of generation terms such as ionization, attachment, etc., and loss terms involving recombination. Electrons and ions gain momentum from the applied electric field and lose momentum due to elastic collisions with the gas. The electron energy equation consists of thermal conduction and convection terms, energy gain from ohmic heating, and energy loss due to a variety of collisional processes. The ions are nearly at the gas temperature since they exchange energy efficiently with the gas molecules. In many respects, these equations and approach are similar to those used in device transport.

In this work, we present results for a helium discharge since it is widely used in processing mixed with reactive gases. The equations were solved in two dimensions to obtain a time-periodic solution in response to the applied frequency of $\omega=13.56 \mathrm{MHz}$. The boundary condition at the powered electrode is 
$\mathrm{V}_{\mathrm{rf}} \sin \omega \mathrm{t}$, where $\mathrm{V}_{\mathrm{rf}}$ is the amplitude of the sinusoidal waveform. The second electrode and the reactor walls are grounded. For a discussion of density, momentum, and energy boundary conditions, reference is made to [1]. The solution procedure starts with constant nominal electron and ion densities as initial conditions and marches in time to obtain a time periodic solution. Typically, convergence requires iterations of thousands of $\mathrm{rf}$ cycles with each cycle divided by at least 100 time steps. This makes the present analysis computationally intensive.

\section{RESULTS AND DISCUSSION}

Figure 1 shows the potential contours within the reactor. The reactor is cylindrically symmetric with two

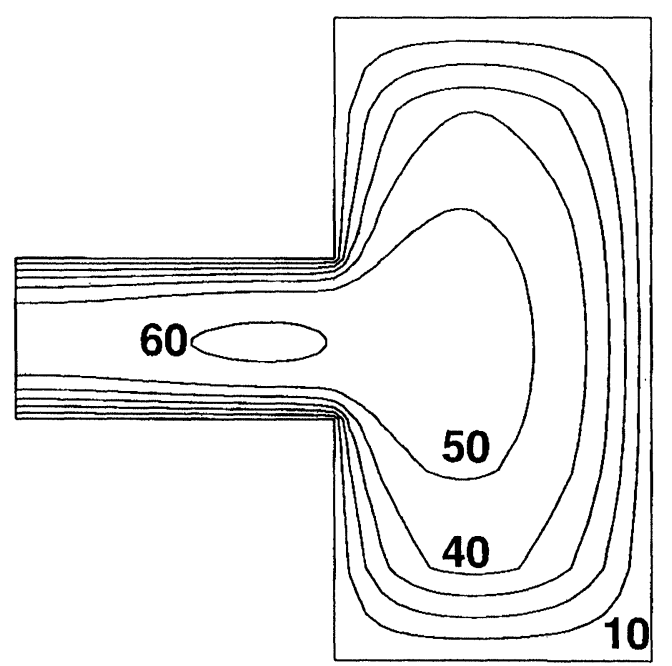

FIGURE 1 Potential contours in a plasma reactor. Contour interval $=10 \mathrm{~V}$. Helium gas at $66.7 \mathrm{~Pa}(500 \mathrm{mTorr})$ and $\mathrm{V}_{\mathrm{rf}}=75 \mathrm{~V}$. Electrode radius $=5.0 \mathrm{~cm}$; electrode separation $=2.5 \mathrm{~cm}$; reactor radius $=10 \mathrm{~cm}$

parallel electrodes, with the lower electrode powered (and contains a wafer) and the upper one grounded. The interelectrode region expands into a side chamber (right side of the figure) at the edge of electrodes. The left boundary in the figures is the line of symmetry. The gas pressure is $500 \mathrm{mTorr}(66.7 \mathrm{~Pa})$ and $\mathrm{V}_{\mathrm{rf}}=75$ $\mathrm{V}$. There is a substantial variation in potential in the radial direction. The radial electrical field is significant near the electrode edges. In the axial direction, the electric field is strong only near the electrodes, since the potential drops significantly in the sheath regions. In the bulk of the plasma, the axial variation in potential is small. Figure 2 shows contours of elec-

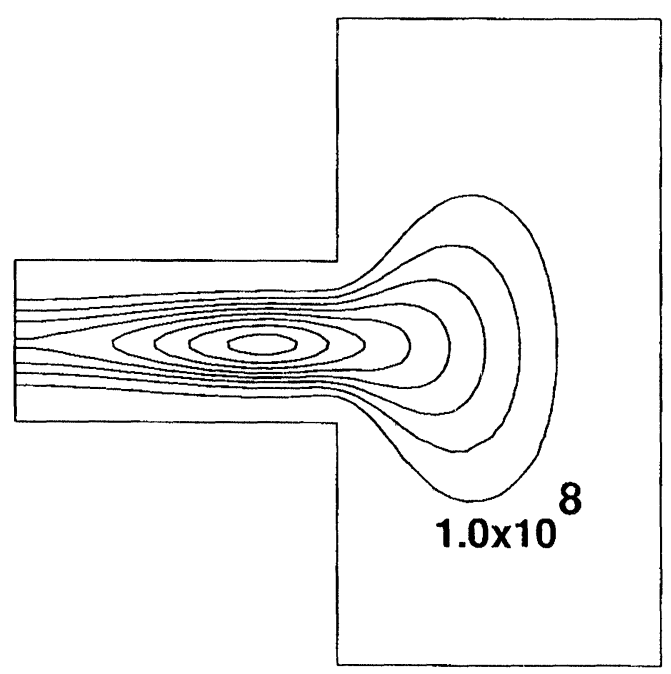

FIGURE 2 Electron density contours Contour interval $=10^{8} \mathrm{~cm}^{-3}$

tron density inside the reactor. It is interesting to see that the maximum in electron density does not occur at the center of the reactor; rather, the maximum is seen off-axis and closer to the electrode edge. The natural tendency of the plasma electrons is to diffuse out toward the reactor walls. However, these electrons experience a strong opposing electric field, as discussed above, forcing them to drift back to the interelectrode region. Consequently, there is a "pile-up" of electrons near the electrode edges. Note that the ion density is only slightly higher in most of the plasma (except the sheaths) since the plasma is almost neutral. Figure 3 shows the electron velocity vectors which serves to illustrate the radially-inward motion of electrons discussed above. Note that near the axis of the reactor, the electron flow is dominantly axial. Ion flux and energy (Figure 4) at the substrate are two quantities of interest since they affect etch/deposition rates and surface quality. Both the flux and energy are not very uniform over most of the substrate. 


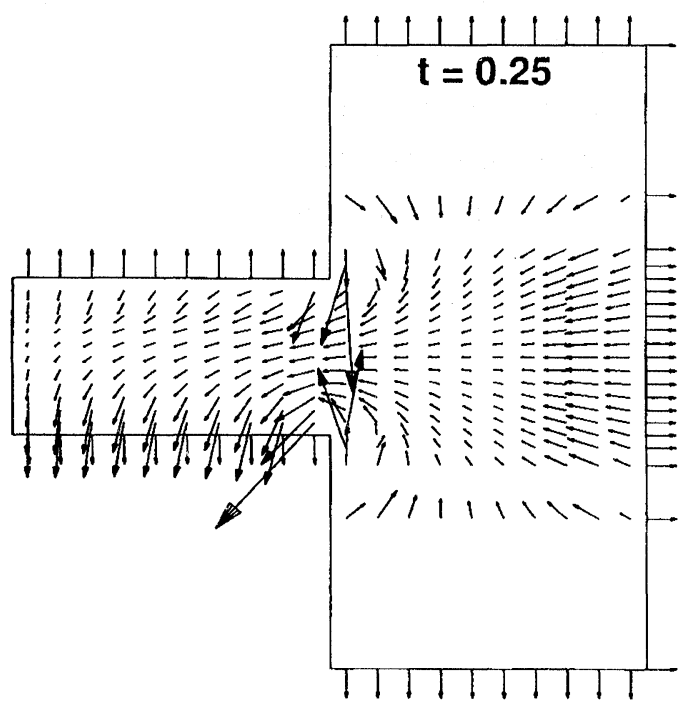

FIGURE 3 Electron velocity vectors

\section{CONCLUDING REMARKS}

In this work, we have discussed two-dimensional plasma simulations in an rf capacitive coupled plasma reactor using a first-principles based model. This type of analysis is computationally very intensive. The computational intensity gets further exacerbated when reactive gas mixtures with multiple ions, radicals and atoms, asymmetric gas injection, and threedimensional effects are included. Then, the present approach is unlikely to be used in CAD efforts; rather, it can serve as a benchmark for validation of more approximate and "quick turnaround" models. The latter may take one of the two following forms. The first approach involves eliminating the need to iterate thousands of rf cycles for time harmonic solution. The plasma properties in the bulk (but not in the sheaths) are essentially invariant during the rf cycle. The massive ions respond only to a time-averaged field. In addition, only time-averaged properties may be ultimately required, since the etch and deposition time scales are orders of magnitude higher than rf time scales. Efforts are in progress to develop such timeaveraged models along with analytical sheath representations. These can be readily integrated with con-

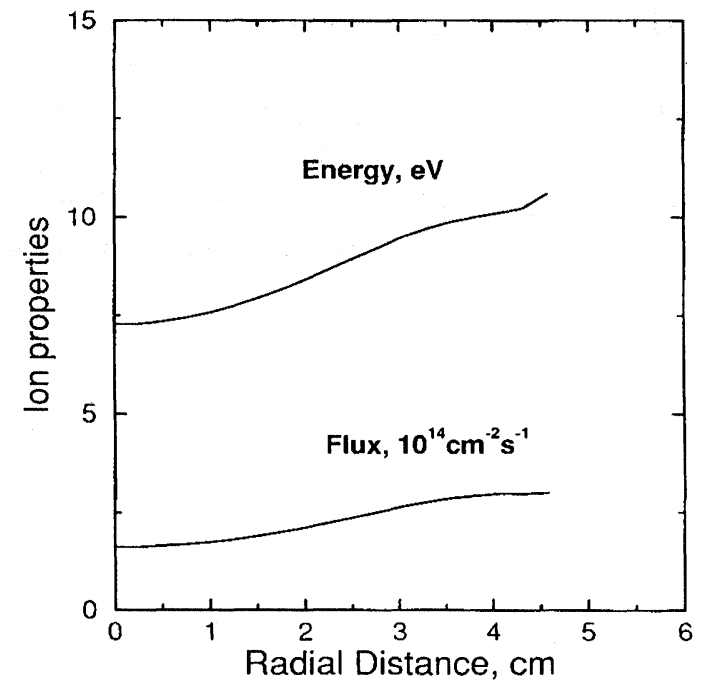

FIGURE 4 Ion flux and energy at the substrate placed on the powered electrode

ventional computational fluid dynamics packages, which then can be used for plasma reactor simulations. The second approach involves making a sacrifice of information on uniformity and other multidimensional effects. Here, one can solve coupled gas flow, energy, and plasma dynamics equations in 0 - or 1-dimension. The 0 -d analysis assumes the reactor contents to be well-mixed. It provides rapid solutions and returns spatially-averaged results as a function of power, pressure, and flow rates. The 1-d model solves radially or axially-averaged governing equations, depending on the reactor configuration. In many cases, the lower order models can provide valuable information using only a small amount of computer time [1]. Efforts are in progress in the modeling community on all approaches discussed above and within a couple of years, CAD-oriented models for plasma reactors must become available.

\section{References}

[1] M. Meyyappan, in Computational Modeling in Semiconductor Processing, M. Meyyappan (Editor), Norwood, MA: Artech House, 1994. 


\section{Biographies}

M. Meyyappan is a Senior Research Scientist at SRA. His research interests include plasma process modeling and computational fluid dynamics in materials processing. He is the editor of a recent book on "Computational Modeling in Semiconductor Processing". He has about twenty-five publications in process and device modeling. T.R. Govindan is a Senior Research Scientist at SRA. His research interests include plasma process modeling, density matrix description of quantum mechanical effects in device physics, and computational fluid dynamics. 

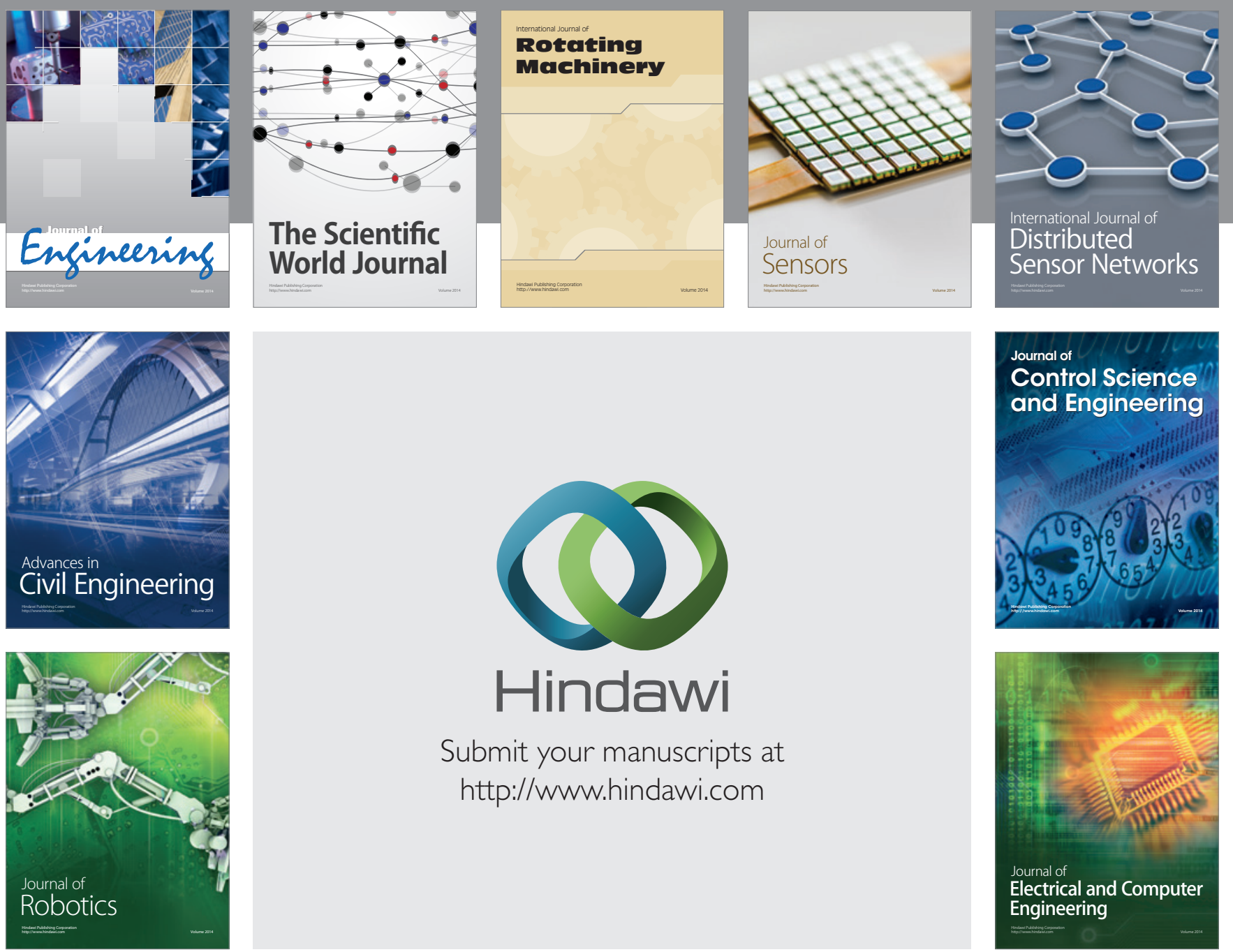

Submit your manuscripts at

http://www.hindawi.com
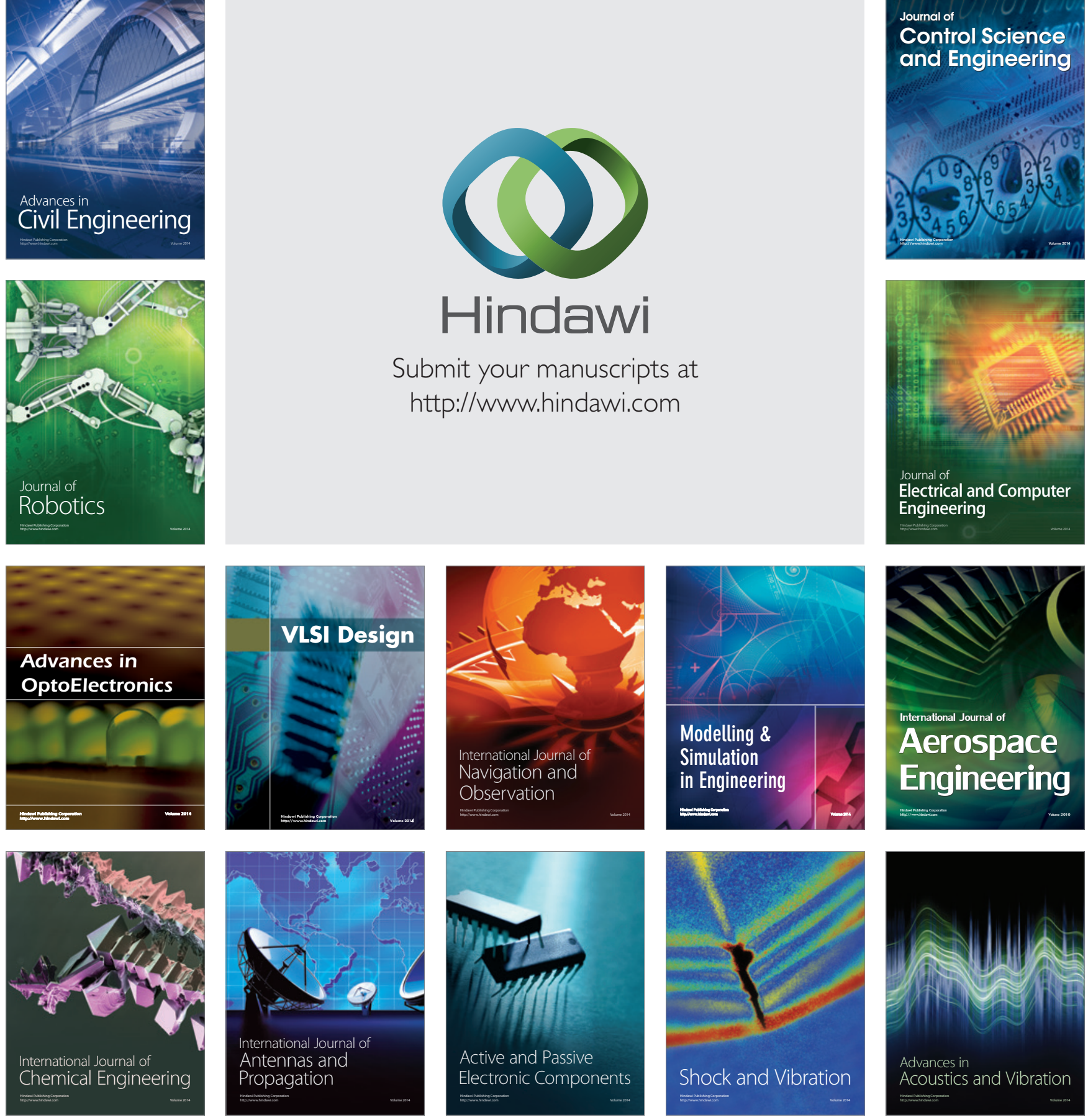